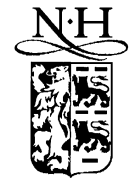

ELSEVIER

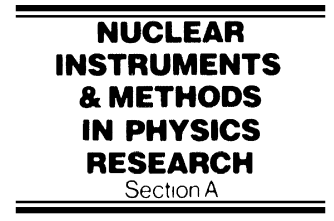

www.elsevier.nl/locate/nima

\title{
Multianode photomultipliers as position-sensitive detectors of single photons
}

\author{
S. Korpar ${ }^{a, b}$, P. Križan ${ }^{a, c}$, R. Pestotnik ${ }^{a, *}$, A. Gorišek ${ }^{a}$, A. Stanovnik ${ }^{a, d}$, \\ M. Stariča ${ }^{\mathrm{a}}$ D. Škrk ${ }^{\mathrm{a}}$

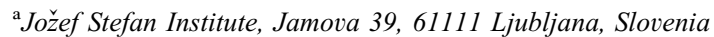 \\ ${ }^{\mathrm{b}}$ Faculty of Chemistry and Chemical Engineering, University of Maribor, Slovenia \\ ${ }^{\mathrm{c}}$ Faculty of Mathematics and Physics, University of Ljubljana, Slovenia \\ ${ }^{\mathrm{d}}$ Faculty of Electrical Engineering, University of Ljubljana, Slovenia
}

\begin{abstract}
Tests of 2300 Hamamatsu multianode photomultipliers (1543 pieces of R5900-M16 and 762 pieces of R5900-M4) have been tested on the bench in order to evaluate their performance for use as position-sensitive photon detectors in the ring imaging Cherenkov counter of the HERA-B experiment. ${ }^{1}$ A weak and stable source of photons has been obtained by Cherenkov radiation of electrons from a ${ }^{90} \mathrm{Sr}$ source in a quartz radiator. From measurements of the count rate versus high voltage, optimal high-voltage values have been obtained as well as a parameter (RQE) corresponding to the relative quantum efficiency. Distributions of the number of photomultipliers as a function of the optimal high voltage and as a function of the relative quantum efficiency are presented. The measured parameters are in good correlation with those provided by the manufacturer. The stability of the photomultipliers has also been followed over two years. The observed count rate decrease is consistent with the radioactive decay of ${ }^{90} \mathrm{Sr}$. (C) 2000 Elsevier Science B.V. All rights reserved.
\end{abstract}

\section{Introduction}

The HERA-B experiment intends to measure CP violation in the $\mathrm{B}$ meson system [1]. The $\mathrm{B}$ mesons are produced by interactions of protons from the $920 \mathrm{GeV}$ beam halo in eight fixed target ribbons. In

\footnotetext{
* Corresponding author.

E-mail address: rok.pestotnik@ijs.si (R. Pestotnik)

${ }^{1}$ The groups responsible for the HERA-B RICH comprise: University of Texas at Austin, University of Barcelona, University of Coimbra, Portugal, Northwestern University, University of Houston, J. Stefan Institute and University of Ljubljana, Slovenia, with support from DESY and the University of Hamburg.
}

order to detect a few thousand of the chosen "goldplated" $\mathrm{B}_{0} \rightarrow \mathrm{J} / \psi K_{\mathrm{s}}^{0} \rightarrow l^{+} l^{-} \pi^{+} \pi^{-}$events in one year of measurement, a reaction rate of $40 \mathrm{MHz}$ is required. With each interaction producing up to 100 charged particles, it is obvious that the detectors will have to cope up with very high rates.

The flavour of the $\mathrm{B}$ meson will be tagged by detecting the charge of the kaon produced upon decay of the associated B meson [1]. Identification of this kaon will be performed by a ring imaging Cherenkov counter (RICH). Initially, a multiwire chamber with CsI photocathode and then a TMAE-based gaseous detector were studied as candidates for photon detection in the RICH. Both had to be abandoned due to deterioration of their 
behaviour in a high-rate environment such as that of HERA-B. At the same time, M4 and M16 R5900 multianode photomultiplier tubes by Hamamatsu became available, so they were chosen for the job $[2,3]$.

Tests of all the 2300 PMTs (1543 M16's and 762 M4's) have been made in the laboratory. On the basis of these tests, the photomultipliers have been grouped according to similar high-voltage characteristics, allowing all PMTs within a group to be connected to the same high voltage, thus maximizing the efficiency for the given, much smaller number of independent HV channels. The results obtained for the optimal high voltage as well as for the relative PMT sensitivity are compared to values provided by the manufacturer. Also the stability of the PMTs has been studied and is presented below.

\section{Experimental apparatus}

The experimental set-up for testing the photomultiplier is shown in Fig. 1. A base board carrying four PMT sockets on one side and resistor chains with amplifier-shaper-discriminator electronics (the ASD8 boards [4]) on the other side, is screwed into an opening in a steel support plate. The photomultipliers are enclosed by a light-tight cylindrical aluminium box carrying a quartz crystal at the top. The crystal is covered by a black, $0.5 \mathrm{~mm}$ thick mylar foil on which the ${ }^{90} \mathrm{Sr}$ source is placed.

The high voltage for the PMTs is supplied by a programmable high-voltage contoller (CAEN N470), controlled by a personal computer through a CAENET card (Fig. 2). The PC also sets the thresholds of the ASD electronics via a GPIB interface (LeCroy 8900A) and a digital-to-analog converter (CAEN C221). The low voltage $( \pm 3 \mathrm{~V})$ for ASD8 boards is supplied by a separate power and supply.

The anode pad signals from the ASD electronics are first converted into ECL and NIM form in a comparator (LeCroy MVL) and ECL-NIM-ECL converter (EG\&G EC1600). The ECL signals are then counted in a 32 channel CA-MAC scaler (LeCroy 4434). From NIM signals an OR and an extended OR $(1 \mu \mathrm{s})$ are formed in a FAN IN/FAN

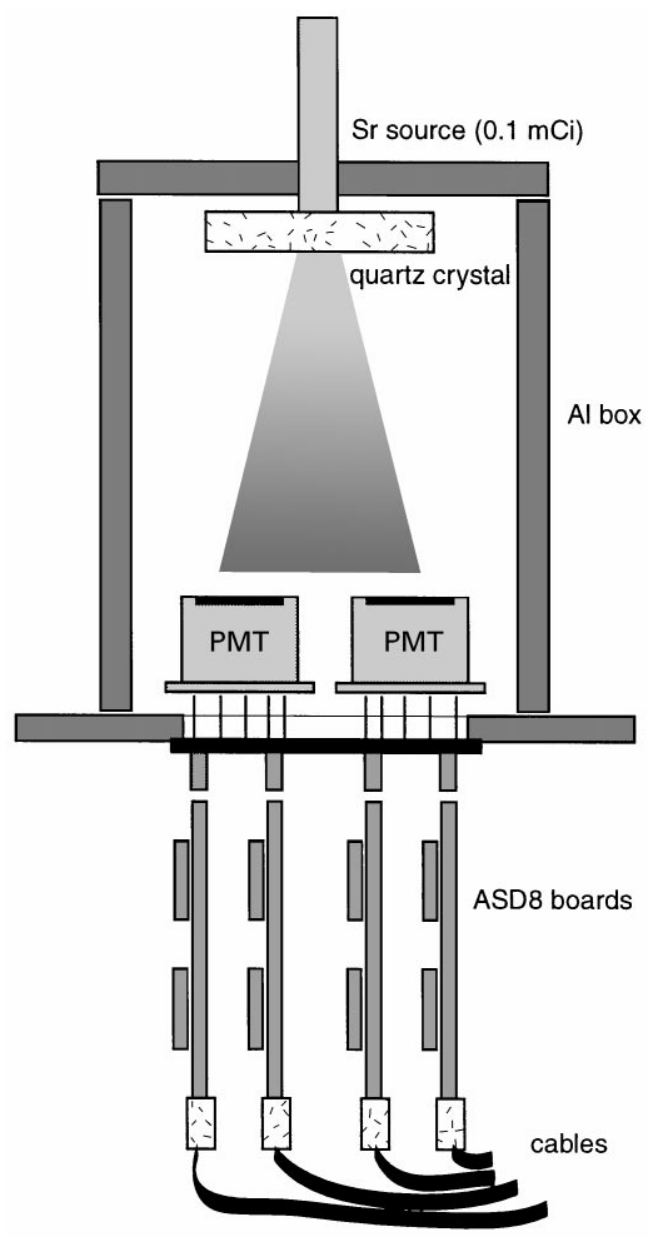

Fig. 1. The experimental set-up.

OUT (EG\&G LF4000) and coincidence (EG\&G CO4000) unit, respectively. Both OR signals are additionally, shaped in a CAMAC discriminator (Philips Scientific 7106) and counted in a eight channel CAMAC scaler (CAEN C257). The inhibit/enable gate is formed by a CAMAC gate generator (CAEN C423).

Besides setting the PMT high voltage and the ASD threshold, the PC also runs a LabWindows data acquisition programme, which runs on the Windows 95 platform. For each photomultiplier, 18 variables (HV or threshold, time, count rates on 16 PMT channels) are written on to an ASCII file, which is then analysed in PAW. 


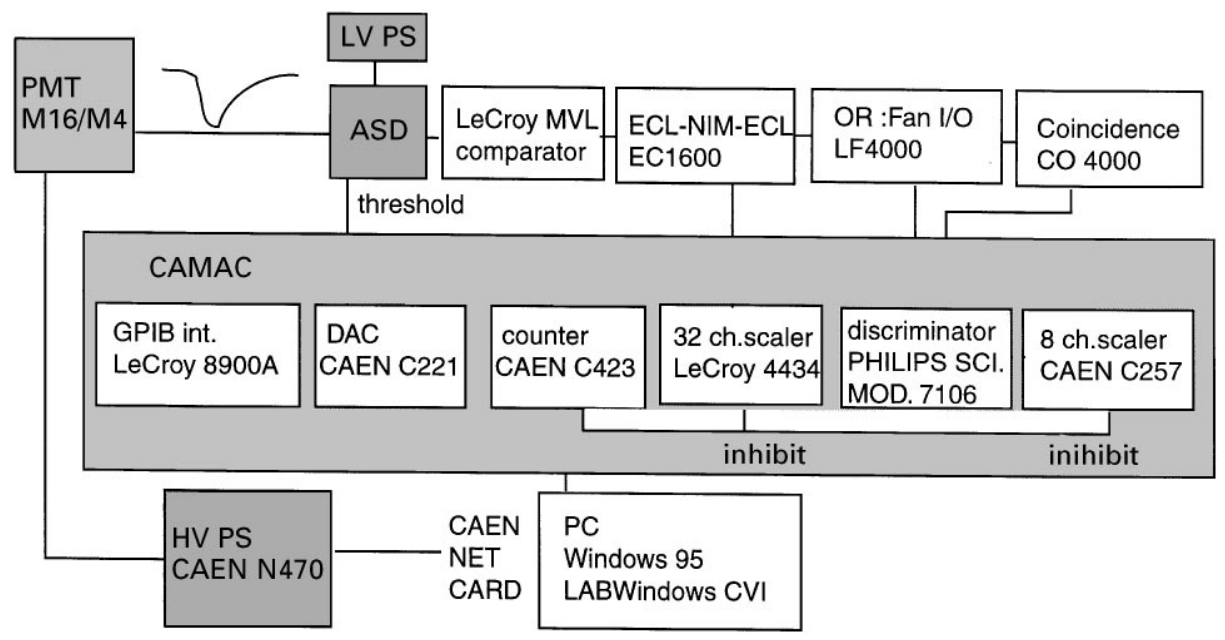

Fig. 2. Block diagram of electronic modules.

The distribution of photons hitting the photomultipliers is not expected to be uniform. A simulation was performed in order to obtain correction factors for the count rates of individual anode pads. The simulation takes into account the ${ }^{90} \mathrm{Sr} \beta$-particle energy distribution and their propagation through the crystal, where they scatter, lose their energy and radiate Cherenkov photons. The photon then reflects or refracts at the crystal surface and finally either hits or misses the photocathodes. The variation of the simulated count rate turned out not to be so large $(20 \%$ difference from the center to the corner of a $2 \times 2$ PMT group).

One of the four PMT sockets was occupied by a reference photomultiplier which was not changed in order to have control over possible count rate variations not arising from quantum efficiency differences. Thus, only 3 PMTs could be measured at a time. The procedure was to insert the 3 PMTs and close the Al box cover. It was found that 15 min of darkness reduced the background count rate to a few counts per second per channel, which is sufficiently low compared to the source count rate of about $1000 \mathrm{~s}^{-1}$ per channel. For a chosen threshold value, a high-voltage scan in steps of $25 \mathrm{~V}$ was automatically started by the computer. Then the ${ }^{90} \mathrm{Sr}$ source was inserted manually and another HV scan in addition to a threshold scan was performed.
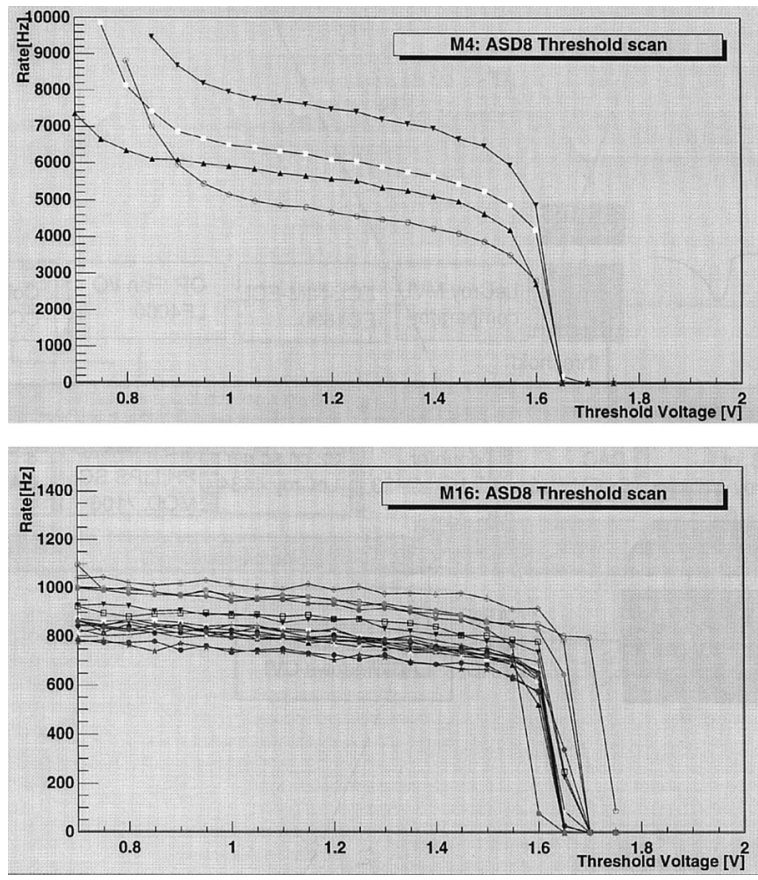

Fig. 3. Dependence of the count rate on the threshold voltage for a typical M4 (above) and a M16 (below) photomultiplier tube.

\section{Results}

A single threshold value was found to be suitable for all photomultipliers (Fig. 3). For each channel of 

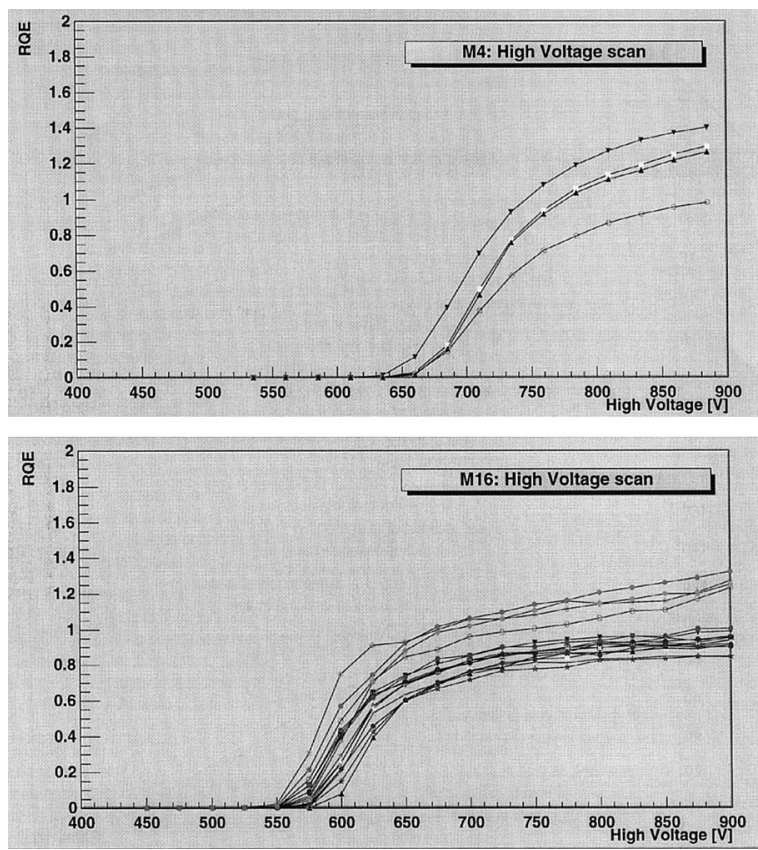

Fig. 4. Dependence of the RQE factor (which is proportional to the count rate-see text) as a function of the high voltage for all the channels of a representative M4 (above) and M16 (below) photomultiplier tube.

each photomultiplier tube, the following characteristic values were determined:

(1) Relative quantum efficiency (RQE), which is just the count rate corrected for non-uniformity of the photon distribution, divided by 10 in the case of M16's and divided by 40 in the case of M4's. For M16 PMTs, the RQE number on the efficiency plateau is thus expected to be about 100 (Fig. 4). Although the division with an extra factor of 4 in the case of M4 PMTs approximately compensates the larger anode pad surface, the RQE numbers on the plateau are still larger by about $50 \%$ (Fig. 4). This is on account of the M4 UV glass window compared to the borosilicate windows on the M16 PMTs.

(2) Recommended or "optimal" high voltage was then defined to be $100 \mathrm{~V}$ above the characteristic voltage at which RQE reaches 40 and 120 for M16's and M4's, respectively.

(3) The background count rate defined as the count rate without the source and after the
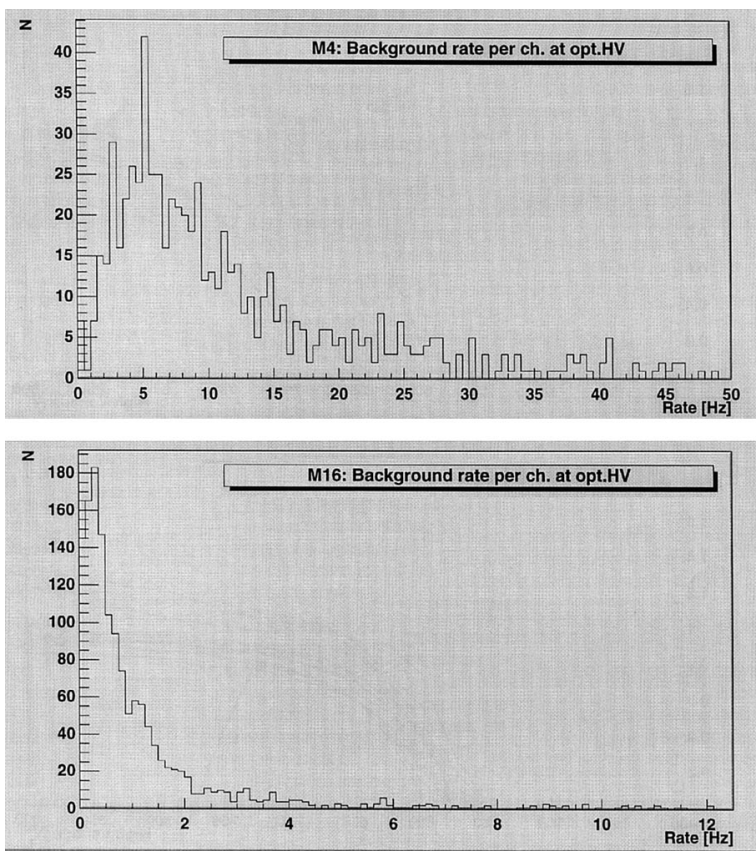

Fig. 5. Distribution of M4 (top) and M16 (bottom) channels depending on their background count rate.
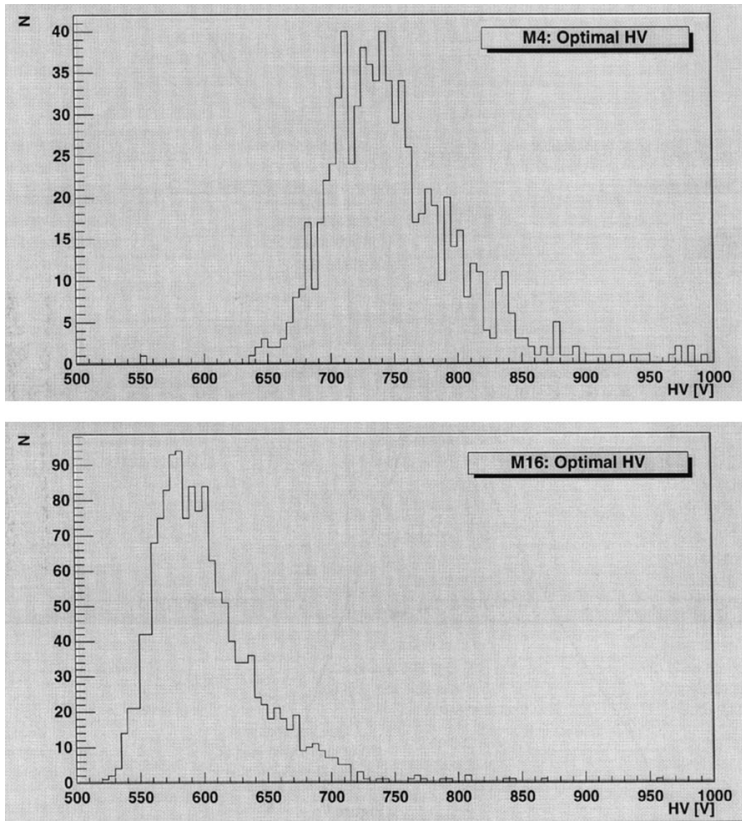

Fig. 6. Distribution of M4 (top) and M16 (bottom) photomultipliers depending on the value of their recommended high voltage. 
PMT has been in darkness for at least 15 min. Fig. 5 gives the distribution of channels as a function of their background count rate. The lower rates for M16's are due to the smaller anode area corresponding to one channel.

Results will be presented as average values over all the channels of one photomultiplier. Fig. 6 gives the distribution of M4 and M16 photomultipliers versus their recommended high-voltage value. This value is seen to be larger for M4's than for M16's, which is a consequence of the smaller number of dynodes (10 for M4's compared to 12 for M16's).

The distributions of photomultipliers depending on their relative quantum efficiencies are shown in Fig. 7 separately for M16's and for M4's. The top two diagrams are the distributions at fixed highvoltage values $(750 \mathrm{~V}$ for M16's and $850 \mathrm{~V}$ for M4's), whereas in the lower two diagrams the RQE values have been taken at the recommended highvoltage values. It is seen that the use of recommended voltages narrows the distributions. Fig. 8 shows the correlation between the recommended high voltage as obtained in this work versus the EBB values given by the manufacturer as corresponding to a gain of $3 \times 10^{5}$. Fig. 9 represents the correlation between the RQE value at the recommended voltage as obtained by us, versus the cathode blue sensitivity or SKB value given by Hamamatsu [3]. Finally, Fig. 10 shows the time dependence of the rate per channel of the reference M4 and M16 photomultipliers. The count rate decrease is consistent with the known decay rate of the ${ }^{90} \mathrm{Sr}$ source.

\section{Conclusions}

After testing 2300 multianode photomultipliers, we conclude that they should meet all the requirements for photon detection in the HERA-B experiment. The measured high-voltage characteristics of all the PMTs allow a HV connecting scheme to be established, according to which several PMTs are connected to the
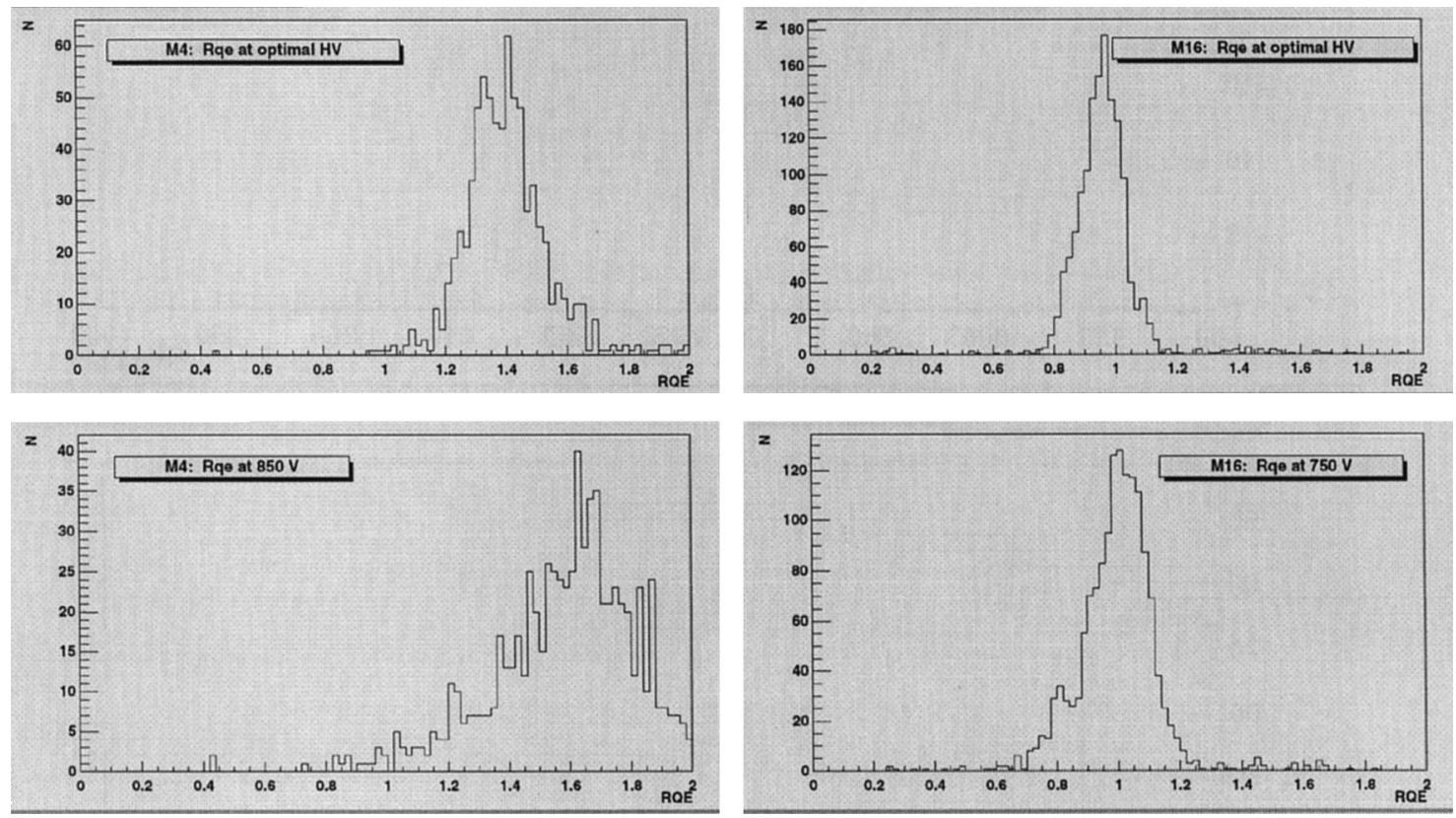

Fig. 7. Distribution of M4 (left) and M16 (right) photomultipliers according to their RQE factor. On the top diagrams, the RQE is taken at a given value of the voltage ( $850 \mathrm{~V}$ for M4's and $750 \mathrm{~V}$ for M16's), while in the lower diagrams the RQE is taken at the recommended voltage of each photomultiplier. 

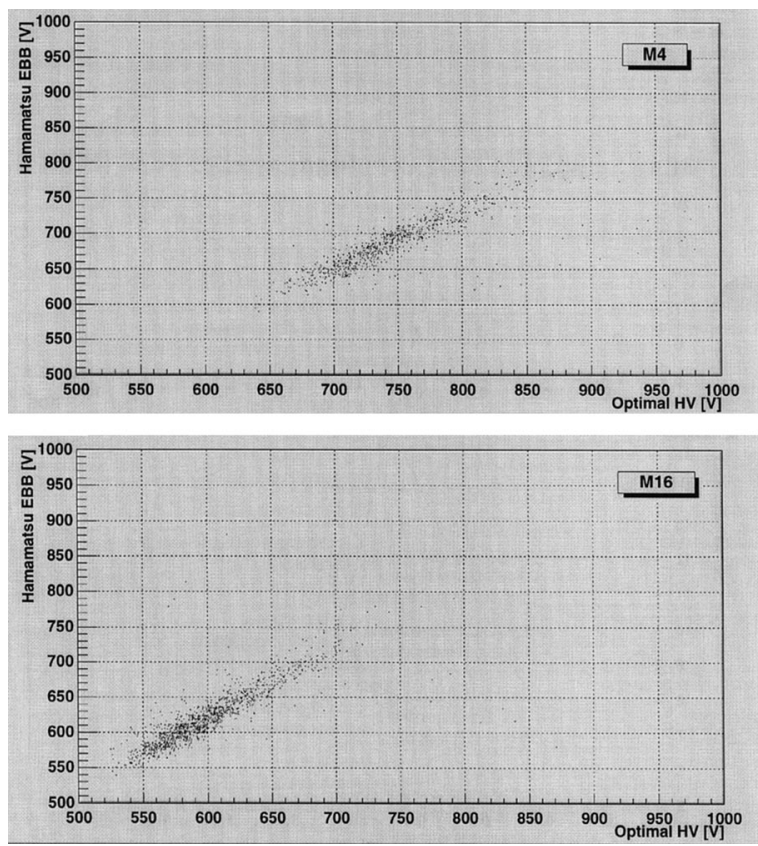

Fig. 8. Correlation between the recommended voltage obtained in this work and the Hamamatsu EBB value corresponding to a gain of $3 \times 10^{5}$. Each photomultiplier is represented by a point with coordinates given by the recommended voltage and the Hamamatsu EBB value. The top diagram is for M4's, the lower diagram is for M16's.
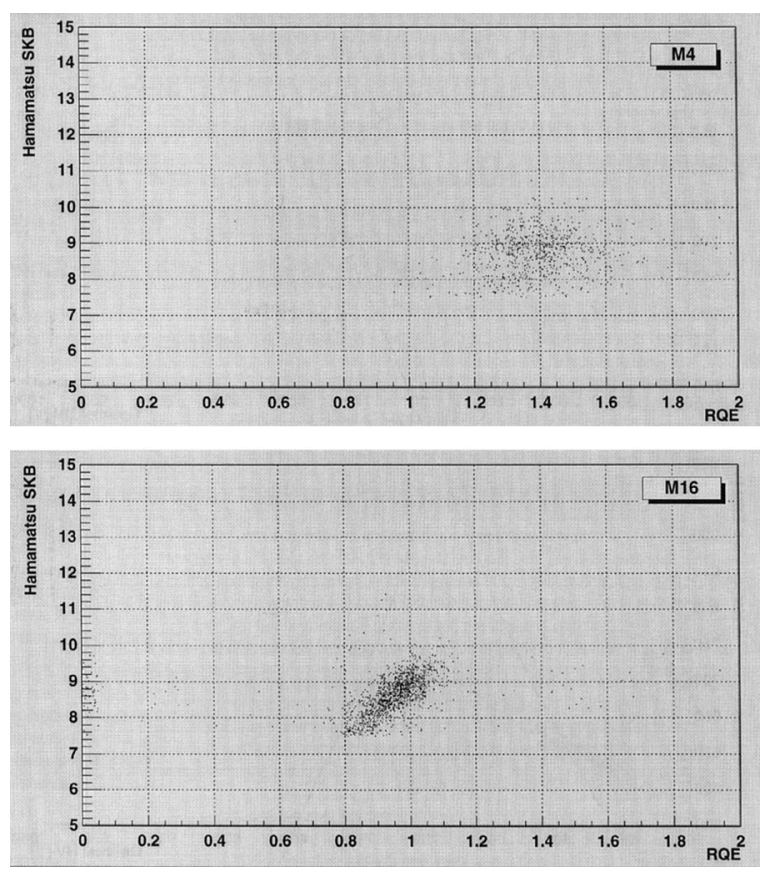
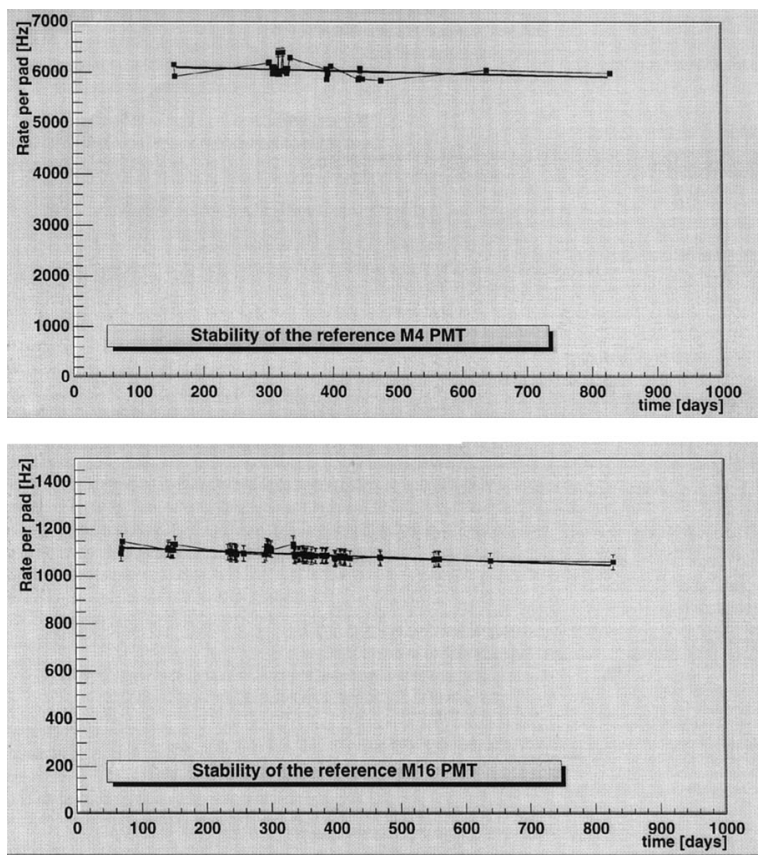

Fig. 10. The time dependence of the count rates of the reference photomultipliers M4 is on top diagram, M16 is on lower one. The curves represent the radioactive decay of ${ }^{90} \mathrm{Sr}$, which thus accounts for the observed count rate decrease.

same HV value without loss of efficiency. The use of recommended $\mathrm{HV}$ values also reduces the dispersion of the sensitivity. In addition, no reduction of performance has been found over longer periods of operation.

\section{References}

[1] T. Lohse et al., Proposal for HERA-B DESY PRC 94/02, 1994.

[2] P. Križan et al., Nucl. Instr. and Meth. A 394 (1997) 27-34.

[3] Hamamatsu Data Sheets for R5900-M16 and R5900-M4 Photomultipliers.

[4] F.M. Newcomer et al., IEEE Trans. Nucl. Sci. 40 (4) (1993) 630.

Fig. 9. Correlation between the relative quantum efficiency (RQE) obtained in this work and the cathode blue sensitivity (SKB) given by Hamamatsu. Each photomultiplier is represented by a point with coordinates given by the RQE value and SKB. The top diagram is for M4's, the lower one is for M16's. 